\title{
New Technologies - New Insights into the Pathogenesis of Hepatic Encephalopathy
}

\author{
Luisa Baker ${ }^{*}$, Bernard Lanz ${ }^{2,3 *}$, Fausto Andreola ${ }^{4}$, Javier Ampuero ${ }^{5,6}$, Anisha \\ Wijeyesekera ${ }^{7}$, Elaine Holmes ${ }^{7}$, Nicolaas Deutz ${ }^{8}$
}

1 Department of Clinical Science and Services, Royal Veterinary College, Hatfield, Hertfordshire, UK

${ }^{2}$ Laboratory for Functional and Metabolic Imaging, Ecole Polytechnique Fédérale de Lausanne (EPFL), Lausanne, Switzerland

${ }^{3}$ Sir Peter Mansfield Imaging Centre, School of Physics and Astronomy, University of Nottingham, University Park, Nottingham, United Kingdom

${ }^{4}$ Liver Failure Group, UCL Institute for Liver and Digestive Health, UCL Medical School, Royal Free Hospital, London, UK

${ }^{5}$ Inter-Centre Unit of Digestive Diseases, Virgen Macarena - Virgen del Rocío University Hospitals, Sevilla, Spain

${ }^{6}$ Instituto de Biomedicina de Sevilla. Sevilla, Spain

${ }^{7}$ Division of Computational and Systems Medicine, Department of Surgery and Cancer, Faculty of Medicine, Imperial College London, London SW7 2AZ, UK.

${ }^{8}$ Department of Health \& Kinesiology, Texas A\&M University, Texas, USA

* Both authors contributed equally to the present work and thus share first authorship.

Running title: New technologies in hepatic encephalopathy

Key words: hepatic encephalopathy, genomics, nuclear magnetic resonance spectroscopy, microRNA, metabonomics, targeted fluxomics

\section{Correspondence to:}

Bernard Lanz

Sir Peter Mansfield Imaging Centre

University of Nottingham, University Park, Nottingham, NG7 2RD, UK

Email: Bernard.Lanz@nottingham.ac.uk 


\begin{abstract}
Hepatic encephalopathy (HE) is a neuropsychiatric syndrome which frequently accompanies acute or chronic liver disease. It is characterized by a variety of symptoms of different severity such as cognitive deficits and impaired motor functions. Currently, HE is seen as a consequence of a low grade cerebral oedema associated with the formation of cerebral oxidative stress and deranged cerebral oscillatory networks. However, the pathogenesis of HE is still incompletely understood as liver dysfunction triggers exceptionally complex metabolic derangements in the body which need to be investigated by appropriate technologies. This review summarizes technological approaches presented at the ISHEN conference 2014 in London which may help to gain new insights into the pathogenesis of HE. Dynamic in vivo ${ }^{13} \mathrm{C}$ nuclear magnetic resonance spectroscopy was performed to analyse effects of chronic liver failure in rats on brain energy metabolism. By using a genomics approach, microRNA expression changes were identified in plasma of animals with acute liver failure which may be involved in interorgan interactions and which may serve as organ-specific biomarkers for tissue damage during acute liver failure. Genomics were also applied to analyse glutaminase gene polymorphisms in patients with liver cirrhosis indicating that haplotype-dependent glutaminase activity is an important pathogenic factor in HE. Metabonomics represents a promising approach to better understand HE, by capturing the systems level metabolic changes associated with disease in individuals, and enabling monitoring of metabolic phenotypes in real time, over a time course and in response to treatment, to better inform clinical decision making. Targeted fluxomics allow the determination of metabolic reaction rates thereby discriminating metabolite level changes in $\mathrm{HE}$ in terms of production, consumption and clearance.
\end{abstract}




\section{Abbreviations}

ALF; $\quad$ acute liver failure

ALI; $\quad$ acute liver injury

APAP; acetaminophen

BDL; bile duct-ligation

CFF; $\quad$ critical flicker frequency

CPP; $\quad$ cerebral perfusion pressure

DAMP; damage-associated molecular pattern molecules

GLS; $\quad$ glutaminase

HCC; hepatocellular carcinoma

HE; hepatic encephalopathy

MHE; minimal hepatic encephalopathy

miRNA; micro RNA

MRS; magnetic resonance spectroscopy

NMR; nuclear magnetic resonance

PHES; psychometric hepatic encephalopathy score

TCA; tricarboxylic acid cycle 


\section{Bernard Lanz: Effects of chronic hepatic encephalopathy on brain energy metabolism, studied by in vivo ${ }^{13} \mathrm{C}$ MRS in rats}

Hepatic encephalopathy is characterized by a wide range of neurological disorders which are cascade events initiated by an increase in blood and brain ammonia resulting from liver failure. However, the molecular basis for the neurological disorders remains unclear and the cause and effect link between the observed disturbances among which changes in amino acids concentrations, energy metabolism dysfunction, alteration of oxidative stress, mitochondrial permeability transition or neurotransmission and molecular transporters activity is still to be determined (Butterworth 2014).

Dynamic ${ }^{13} \mathrm{C}$ nuclear magnetic resonance spectroscopy (MRS) is one of the numerous in vivo applications of NMR, which is of particular interest for probing brain energy metabolism in a non-invasive way (de Graaf et al. 2003; Gruetter 2002; Henry et al. 2006; Lanz et al. 2013b; Mason and Rothman 2004). ${ }^{13} \mathrm{C}$ is a stable isotope of carbon present with a natural abundance of $1.1 \%$ and has a non-zero nuclear magnetic moment, which makes it an NMR active nucleus. On the other hand, the most abundant carbon isotope ${ }^{12} \mathrm{C}(\mathrm{NA}=98.9 \%)$ has a zero nuclear magnetic moment and therefore cannot be detected through NMR. This characteristic can be exploited to probe metabolic processes in vivo by infusing ${ }^{13} \mathrm{C}$-enriched substrates into the studied subject and following the incorporation of the ${ }^{13} \mathrm{C}$ atoms into the metabolic products.

Following previous ex vivo ${ }^{13} \mathrm{C}$ MRS studies (Bosoi et al. 2014), the presented work illustrates the possibilities of in vivo dynamic ${ }^{13} \mathrm{C}$ MRS on a bile duct-ligated (BDL) rat model to identify how at later stages of chronic liver disease, increased brain glutamine impacts brain oxidative metabolism and glutamate/glutamine cycling.

The nowadays availability of horizontal high magnetic field MRS scanners for in vivo applications, coupled with strong magnetic field gradients and refined methods for magnetic field homogenization (Gruetter and Tkac 2000) enables the measurement of well-resolved NMR spectra with temporal resolutions suitable for the analysis of biochemical processes involved in oxidative metabolism and neurotransmission (Lanz et al. 2013a) (Fig. 1A). In 
particular for ${ }^{13} \mathrm{C}$, the increased sensitivity obtained at high fields such as $9.4 \mathrm{~T}$ enables the measurement of the ${ }^{13} \mathrm{C}$ enrichment turnover of the $\mathrm{C} 4, \mathrm{C} 3$ and $\mathrm{C} 2$ positions of glutamate and glutamine, resulting from oxidative metabolism of labelled substrates such as [1,6${ }^{13} \mathrm{C}_{2}$ ]glucose in astrocytic and neuronal tricarboxylic acid (TCA) cycles, as well as carbon chain exchanges in glutamatergic neurotransmission processes. The analysis of this dynamic ${ }^{13} \mathrm{C}$ enrichment using dedicated metabolic models, such as the two-compartment neuronal/glial previously applied in this field (Gruetter et al. 2001; Lanz et al. 2013b) enables the astrocytic and neuronal TCA cycle fluxes, transmitochondrial exchange with cytosolic amino acids as well as apparent glutamatergic neurotransmission and astrocytic anaplerosis.

In the present study, eight weeks after surgery, male Wistar rats ( $n=7$ BDL rats, $n=5$ sham operated rats) were intravenously infused with $\left[1,6-{ }^{13} \mathrm{C}_{2}\right]$ glucose under isoflurane anaesthesia and in vivo ${ }^{13} \mathrm{C}$ MRS was undertaken over 5 hours at 9.4T, using a semi-adiabatic adaption of DEPT polarization transfer for in vivo applications (Henry et al. 2003). This sequence enables the localized measurement (320 $\mu 1$ voxel) of a wide range of ${ }^{13} \mathrm{C}$-labeled metabolites resulting from glucose metabolism, with the simultaneous advantage of the wide chemical shift range of ${ }^{13} \mathrm{C}$ spectra, the precise location based on the low chemical shift ranges of the coupled ${ }^{1} \mathrm{H}$ nuclei and the increased measurement sensitivity obtained through ${ }^{1} \mathrm{H}-{ }^{13} \mathrm{C}$ polarization transfer (Fig. 1B). In particular, the ${ }^{13} \mathrm{C}$ enrichment turnover of the $\mathrm{C} 4, \mathrm{C} 3$ and C2 positions of glutamate and glutamine were measured in terms of fractional enrichment with a time resolution of 10 minutes (Fig. 1C). For this purpose, total glutamate and glutamine concentrations were determined by ${ }^{1} \mathrm{H}$ MRS prior to the glucose infusion, in the same voxel. This represents the first in vivo dynamic ${ }^{13} \mathrm{C}$ MRS measurement of brain oxidative metabolism in a chronic liver disease model and will further enable to characterize the modifications of the related metabolic fluxes under chronic liver disease (Lanz B., unpublished results). 


\section{Luisa Baker and Fausto Andreola: Increase in plasma levels of brain specific microRNA-124-1 associates with falling cerebral perfusion pressure in an acetaminophen-induced porcine model of acute liver failure.}

Acute liver failure (ALF) is characterized by massive hepatocyte necrosis resulting in release of damage-associated molecular pattern molecules (DAMPs), the ensuing inflammatory response and multi-organ failure, notably of the kidney and brain (O'Grady et al. 1993). MicroRNAs (miRNAs) are short (ca. 18-25 nucleotides), non-coding stands of RNA that function as post-transcriptional regulators by binding to the 3' untranslated region of specific target mRNAs, resulting in repression of translation by promoting mRNA degradation or blocking translation (Bartel 2009). MiRNAs are released into the circulation in various forms, such as inside exosomes, microvesicles, high-density lipoprotein, apoptotic bodies and with proteins (Ago2), and may mediate both short range (cell to cell) and distant (to other organs) communication (Szabo and Bala 2013). MiRNAs not released into the circulation as naked strands of RNA have been shown to be stable in blood samples, making them good candidates for biomarkers (Mitchell et al. 2008).

In the described study (Baker et al. 2015) a clinically relevant porcine model of acetaminophen (APAP)-induced ALF (Lee et al. 2013) was used to investigate temporal changes in both global miRNA and tissue-specific miRNAs in plasma samples from induction of ALF, through acute liver injury (ALI) and ALF to death. Six pigs received oral APAP with ALF occurring after $19 \mathrm{~h} \pm 2 \mathrm{~h}$ and death after a further $13 \mathrm{~h} \pm 3 \mathrm{~h}$, whilst three control pigs underwent the same protocols except for receiving water instead of APAP and were maintained for $20 \mathrm{~h}$ up to 'ALF' and 20h thereafter (Fig. 2A). The study showed significantly increased plasma global miRNA levels in the APAP animals starting at $4 \mathrm{~h}$ before the point of ALF $(P<0.0001)$ and continuing until death, which associated with worsening of clinical markers of ALF progression $(P<0.0001)$; international normalized ratio (INR) (fixed effect 
$(\beta): 1.904$, confidence interval $(\mathrm{CI}): 1.430-2.378)$, creatinine ( $\beta$ : 3.323, CI: $2.769-3.878)$ and cerebral perfusion pressure (CPP) ( $\beta$ : -0.080, CI: -0.096 - -0.065).

To allow quantification of circulating levels of specific miRNA species, which may have been contributing to the observed rise in global miRNA levels, miR26a was identified as a robust endogenous control. Plasma levels of three specific miRNA species, miR122, miR192 and miR124-1, were quantified. Liver-specific miR122 increased significantly in the plasma around the onset of $\operatorname{ALF}(P<0.0001)$ and associated with increasing INR $(P<$ 0.0001, $\beta$ : 3.655, CI: 2.085-5.225). Plasma levels of kidney-enriched miR192 increased significantly $8 \mathrm{~h}$ after $\operatorname{ALF}(P<0.0001)$ and associated with increasing creatinine $(P<0.0001$, $\beta:$ 3.631, CI: 2.329-4.933). Finally circulating brain-enriched miR124-1 increased significantly pre-terminally $(P<0.0001)$, shortly after plasma ammonia increased and at the same time as increasing intracranial pressure and in association with falling $\mathrm{CPP}(P<0.002$, $\beta:-0.041, \mathrm{CI}:-0.066--0.017)$.

To assess whether miRNA were released as a signalling complex or as a result of tissue injury, miRNA levels were analysed in isolated plasma exosomes and showed only low levels of miRNA that were unaltered by progression of ALF, whilst plasma levels of genomic DNA, a DAMP, were significantly elevated from ALF until death $(P<0.0001)$ and associated with plasma global miRNA levels $(P<0.0001, \beta$ : 0.613, CI: 0.506-0.719).

The study concluded that miRNA were released into the plasma as a result of tissue injury with global miRNA levels increasing first. Specific miRNA species revealed a timeline of organ injury with miR122, miR192 and miR124-1 representing liver, kidney and brain injury respectively (Fig. 2B). Future studies, with a suitable human population, should investigate the prognostic potential of global miRNA and tissue-specific miRNA species as biomarkers in patients with ALI and ALF. 


\section{Javier Ampuero: Application of Genomics in Hepatic Encephalopathy}

Genetic tests can be a helpful tool for clinicians to diagnose liver diseases. This is particularly true for monogenic diseases. However, for many liver disorders including those outcomes derived from liver cirrhosis, multiple genes contribute to the risk of the disease by a combination of their phenotypic effects. Moreover, the disease predispositions are multifactorial. Most common hepatobiliary liver diseases and traits are complex disorders and the level of risk depends on multi-dimensional interactions between genetic and environmental factors, which have to be identified. Therefore, refined genome analyses are necessary to clarify the link between a typical array of genes with altered expression and the risk and severity of HE and further enable the design of polygenic disease predisposition tests.

Hepatic encephalopathy (HE) is a major complication of liver cirrhosis with an array of clinical manifestations and comprises a spectrum of neuropsychiatric abnormalities (Romero-Gomez and Ampuero 2014). HE is graded according to the severity of the symptoms. The spectrum of HE encompasses patients with no clinical alterations beyond of impairments in neuropsychological and neuropsychiatric tests (minimal HE, (MHE)), patients with low-grade or covert HE including MHE and overt HE grade 1, and patients with overt HE (typically with grades 2, 3 and 4). West Haven criteria and Glasgow coma scale are typically used to determine overt HE, while patients with MHE could be overlooked if not systematically investigated with higher sensitivity tests.

A wide range of tests have been used to determine the mental status in patients with liver cirrhosis (Romero-Gomez and Ampuero 2014), including psychometric (psychometric hepatic encephalopathy score, PHES), neuropsychological (critical flicker frequency, CFF) and computerized tests, which explore cognition, behaviour, biologic regulation and emotion. Using these tools, different neuropsychological stages have been related to MHE, without being entirely specific. Recently, Kircheis et al. (Kircheis et al. 2014) explored the usefulness of CFF and PHES in the diagnosis of low-grade HE and illustrated the usefulness of CFF in identifying various stages within the spectrum of HE. All these complementary methods help to identify patients at risk of suffering overt HE, falls, traffic accidents and impaired quality of 
life. The results of these tests show a continuum with respect to the severity of HE and require therefore the establishment of specific thresholds to define MHE, which remain controversial.

$\mathrm{HE}$ is resulting from interorgan interactions and systemic hyperammonia plays a major role in the pathogenesis of HE (Romero-Gomez et al. 2004). The source of this ammonia remains unclear. One possible pathway is glutamine utilization as an energy source by the intestinal cells. A genetic factor influencing on the development of overt HE has been identified based on the length of alleles (short vs. long) of a microsatellite in 5'UTR promoter region of the phosphate-activated glutaminase (GLS) gene and on the presence of TACC haplotype (Romero-Gomez et al. 2010). In the current study, patients with cirrhosis were divided in two groups based on the presence of TACC haplotype of the glutaminase gene. PHES as well as CFF tests (Kircheis et al. 2002; Romero-Gomez et al. 2007) were used as MHE evaluation methods. The genetic analyses showed that patients harboring non-TACC haplotype had significantly lower events of overt HE with normal CFF results in comparison with those with altered CFF, supporting the role of variant GLS in HE development. On the

other hand, the TACC haplotype was associated with the absence of overt hepatic encephalopathy, irrespective of the result of CFF. This study showed that glutaminase activity plays a major role on the overt HE and could influence on the MHE tests (Fig. 3). Evaluation of GLS gene should be incorporated to clinical practice as additional screening method.

\section{Anisha Wijeyesekera and Elaine Holmes: Application of Metabonomics: The Role of Metabolic Profiling in Systems Medicine}

Hepatic encephalopathy is characterized by metabolic derangements, the study of which is important to our understanding of the disease process and for the discovery of novel treatment options. Metabonomics (defined as the quantitative measurement of time-related multi-parametric metabolic responses of multicellular systems to pathophysiological stimuli or genetic modification (Nicholson et al. 1999)) offers a unique opportunity to better understand HE, by capturing the systems level metabolic changes associated with disease. Metabolic profiles can identify both endogenous and exposomic (e.g. drug, food and 
microbial) metabolites in any biofluid or tissue using a combination of nuclear magnetic resonance (NMR) spectroscopy and mass spectrometry (MS), often hyphenated with preseparation techniques such as gas chromatography, ultra performance liquid chromatography and capillary electrophoresis. Multivariate pattern recognition analysis of the spectra allows identification of chemical signatures associated with disease class or condition, and further statistical correlation allows identification of other metabolites that are correlated with a particular marker. This data can then be interpreted by relating it to metabolic pathways and integrating it with complementary omics data. Once marker metabolites of interest have been identified, a targeted metabolite assay is developed to capture and quantify those metabolites.

The potential applications of metabonomics to liver disease research are diverse and the following are examples of this work. A recent study applied an untargeted metabolic screening approach using NMR spectroscopy to characterise the metabolic phenotype of patients with or without cirrhosis, and with or without HE compared to gender and age matched healthy controls. The results revealed an increase in lactate and five amino acids in HE patients compared with controls; these metabolites are precursors for gluconeogenesis and indicative of impaired energy production. Furthermore, ketone bodies were also elevated in cirrhotic and HE patients compared with controls; acetoacetate was observed to be higher in HE compared to cirrhosis (in comparison with the other ketone bodies $\beta$-hydroxybutyrate and acetate). The authors hypothesise that these findings are indicative of impaired ketogenesis in HE (Dabos et al. 2015). Another study using a similar approach identified changes in amino acid and lipid metabolites in plasma from patients with liver fibrosis compared to healthy controls (Sands et al. 2015). These changes were indicative of impaired aromatic amino acid metabolism, impaired methionine metabolism and altered lipid metabolism with liver fibrosis. Metabolic profiling has also been used to investigate the possibility of differentiating hepatocellular carcinoma (HCC) from cirrhosis by analysis of urinary metabolites in order to allow identification and early treatment of patients with $\mathrm{HCC}$ in West Africa, where hepatitis 
B and C prevalence is high (Ladep et al. 2014). The study identified six urinary metabolites; acetylcarnitine, betaine aldehyde, carnitine, creatine, ribitol and $N$-acetylglutamate, which were increased $(P<0.005)$ with presence and grade of HCC compared to cirrhosis. In another application, a toxicological study investigated the effects of the gut microbiome on metabolism of the steatotic hepatotoxin, hydrazine in conventional and germ free rats to explore the role of the gut microbiota in mediating liver toxicity (Swann et al. 2009), and showed an altered urinary metabolite time trajectory in germ free rats corresponding with more severe toxicity and extreme outliers in brain tissue metabolites with severe toxic reactions.

Direct in clinic applications of metabonomics include both longitudinal modelling from patient admission through treatment to outcome and real-time modelling from samples collected at surgery (Kinross et al. 2011). Real-time surgical diagnostics is being investigated through the use of the intelligent knife (iKnife), a cutting electrocautery head attached to a handle that can sample the tissue smoke and run it through a specialized mass spectrometer, which in real-time compares the spectrum of the glycerophospholipids to a tissue specific database and provides the surgeon with feedback as to the tissue type being cut through. The iKnife is currently undergoing clinical trials but early data suggest good success with margins around cancerous tissue (Balog et al. 2013). Finally next generation technologies combining metabonomics and imaging include using matrix-assisted laser desorption/ionization-MS to form topographic chemistry images (Fonville et al. 2013) and using desorption electrospray ionisation imaging on frozen tissue sections to identify tissue types within the section based on co-localisation of optical and metabolite data for each pixel providing a pathway for augmenting clinical decision-making (Abbassi-Ghadi et al. 2014; Mirnezami et al. 2014).

\footnotetext{
Nicolaas Deutz: Targeted fluxomics in humans to measure the synthesis and breakdown of biological molecules.
} 
Protein and amino acid metabolism are important to the pathogenesis of hepatic encephalopathy and the study of changes in metabolite flux during hepatic disease allows better understanding of this pathogenesis. Targeted fluxomics involves the measuring of specific metabolites at the whole body and organ level to determine metabolic reaction rates. Some products that appear in plasma are mainly derived from certain tissue and as such the whole body appearance can be used to estimate organ metabolism. A good example is the appearance of the amino acid 3-methylhistidine that is product in muscle tissue. When it is possible to collect blood across organs or organ tissue like muscle or brain, intracellular metabolism can be estimated.

Several methods have been described to measure the whole body metabolic reaction rates. We and others have used the primed constant and continuous infusion protocol with tracers of substrates to measure the production of the tracee of the substrate (Rate of appearance (Ra)). This approach was also used to measure the conversion of a substrate into a product. A well-known metabolic pathways that is measured with this approach is the conversion of the amino acid phenylalanine into tyrosine. If it is possible to collect blood samples across organs like brain, muscle, kidney, gut or liver, intracellular metabolism in these organs can be estimated.

A primed constant infusion with stable isotopic amino acid tracers followed by a protein meal containing oral isotopes allows calculation of protein synthesis and breakdown, and splanchnic extraction. In patients with chronic obstructive pulmonary disease, splanchnic extraction following a meal was lower than for healthy controls, net protein synthesis was similar for patients and controls relating to the level of available essential amino acids, however patients were more anabolic during the meal than controls (Jonker et al. 2014).

Protein digestibility can be measured by combining a fully labelled protein such as ${ }^{15} \mathrm{~N}$-spirulina with labelled amino acids in a meal and comparing the ratio of the two in the meal to the ratio of the two in blood samples (Engelen et al. 2014) whilst absorption can be assessed by feeding D-amino acids, which are absorbed at the same rates as L-amino acids, 
but cannot be utilized for protein synthesis and therefore escape the gut and liver to be measured in plasma samples.

Fractional protein synthesis and fractional protein breakdown in tissues like brain and muscle can be measured using pulsed infusions of different labelled isotopes of the same amino acid followed by a single tissue biopsy (Tuvdendorj et al. 2014).

Pulses of stable isotopes can provide the same information about whole body protein synthesis and breakdown in the extracellular pool as a primed constant infusion as well as allowing modelling of changes in the intracellular pool (Engelen et al. 2014). In addition by using combinations of different isotopes in a pulse not only can protein synthesis and breakdown be measured, but also nitric oxide, serotonin and glutathione production, myofibrillar and collagen protein breakdown, and transamination and reanimation of various amino acids.

An alternative method for measuring protein synthesis and breakdown in tissue samples like muscle and brain is a single intake of heavy water $\left(\mathrm{D}_{2} \mathrm{O}\right)$, which will label fatty acids and triglycerides as well as certain amino acids, giving an indication of both fat and protein metabolism amongst other things (Wilkinson et al. 2015).

We applied some of the described in models of hepatic failure or in humans with liver disease. The changes seen in metabolite concentrations during liver disease need to be considered in terms of production, consumption and clearance. For instance, we found that plasma arginine concentrations fall in a porcine model of acute liver failure due to increased plasma clearance rather than a fall in arginine production (Sharma et al. 2012). Also, blood in the stomach, as during a gastric bleed, is eating a protein that lacks the essential amino acid, isoleucine. By adding isoleucine enterally or intravenously, will increase liver protein synthesis and by that reduce the nitrogen load of that blood meal (Olde Damink et al. 1997). We found that using transorgan studies of ammonia flux in patients with liver cirrhosis that the portal drained viscera (primarily small intestine) and the kidney produce ammonia whilst the liver and muscle remove ammonia from the circulation (Olde Damink et al. 2002). Organ specific data can enable better, more targeted approaches, such as showing that cooling both 
reduces uptake of ammonia and glutamine production in the brain (Jalan et al. 2004) and that a simulated blood meal stimulates renal ammonia production (Olde Damink et al. 2003).

In more recent studies, we used infusions of stable isotopes of metabolites related to the compound of interest, for example nitric oxide production has been shown to be reduced in septic patients and a stable isotope arginine infusion in sepsis stimulated nitric oxide production and reduced protein breakdown (Luiking et al. 2015; Luiking et al. 2009). We applied the acute pulse method with labelled phenylalanine or the chronic intake of $\mathrm{D}_{2} \mathrm{O}$ measure protein synthesis in organs like brain (several different parts), liver, gut (different parts), muscle etc of rats with chronic bile duct ligation (unpublished Rose et al, 2016). We used the phenylalanine constant infusion protocol to measure muscle protein synthesis in humans with liver cirrhosis (Tsien et al. 2015).

In conclusion, the use of stable tracers will advance the knowledge of the disturbed pathways during liver failure in animals and humans.

\section{Acknowledgements}

The authors warmly thank Dr Boris Görg for his role as writing coordinator for this review article. Bernard Lanz gratefully acknowledges Dr Cristina Cudalbu, Dr Valérie McLin, Dr

Olivier Braissant and Dr Rolf Gruetter for their contributions in the ${ }^{13} \mathrm{C}$ MRS project. The ${ }^{13} \mathrm{C}$ MRS study was supported by Centre d'Imagerie BioMédicale (CIBM) of the UNIL, UNIGE, HUG, CHUV, EPFL, the Leenaards and Jeantet Foundations and SNF grant 131087. 


\section{References}

Abbassi-Ghadi N et al. (2014) Discrimination of lymph node metastases using desorption electrospray ionisation-mass spectrometry imaging Chemical communications 50:3661-3664 doi:10.1039/c3cc48927b

Baker LA et al. (2015) Circulating microRNAs Reveal Time Course of Organ Injury in a Porcine Model of Acetaminophen-Induced Acute Liver Failure PloS one 10:e0128076 doi:10.1371/journal.pone.0128076

Balog $\mathrm{J}$ et al. (2013) Intraoperative tissue identification using rapid evaporative ionization mass spectrometry Science translational medicine 5:194ra193 doi:10.1126/scitranslmed.3005623

Bartel DP (2009) MicroRNAs: target recognition and regulatory functions Cell 136:215-233 doi:10.1016/j.cell.2009.01.002

Bosoi CR, Zwingmann C, Marin H, Parent-Robitaille C, Huynh J, Tremblay M, Rose CF (2014) Increased brain lactate is central to the development of brain edema in rats with chronic liver disease Journal of hepatology 60:554-560 doi:10.1016/j.jhep.2013.10.011

Butterworth RF (2014) Pathophysiology of brain dysfunction in hyperammonemic syndromes: The many faces of glutamine Molecular genetics and metabolism 113:113-117 doi:10.1016/j.ymgme.2014.06.003

Dabos KJ, Parkinson JA, Sadler IH, Plevris JN, Hayes PC (2015) (1)H nuclear magnetic resonance spectroscopy-based metabonomic study in patients with cirrhosis and hepatic encephalopathy World journal of hepatology 7:1701-1707 doi:10.4254/wjh.v7.i12.1701

de Graaf RA, Mason GF, Patel AB, Behar KL, Rothman DL (2003) In vivo ${ }^{1} \mathrm{H}-\left[{ }^{13} \mathrm{C}\right]-\mathrm{NMR}$ spectroscopy of cerebral metabolism NMR in biomedicine 16:339-357

Engelen MP, Com G, Anderson PJ, Deutz NE (2014) New stable isotope method to measure protein digestibility and response to pancreatic enzyme intake in cystic fibrosis Clinical nutrition 33:1024-1032 doi:10.1016/j.clnu.2013.11.004

Fonville JM et al. (2013) Hyperspectral visualization of mass spectrometry imaging data Analytical chemistry 85:1415-1423 doi:10.1021/ac302330a

Gruetter R (2002) In vivo 13C NMR studies of compartmentalized cerebral carbohydrate metabolism Neurochemistry international 41:143-154

Gruetter R, Seaquist ER, Ugurbil K (2001) A mathematical model of compartmentalized neurotransmitter metabolism in the human brain American journal of physiology 281:E100-112

Gruetter R, Tkac I (2000) Field mapping without reference scan using asymmetric echoplanar techniques Magn Reson Med 43:319-323

Henry PG et al. (2006) In vivo ${ }^{13} \mathrm{C}$ NMR spectroscopy and metabolic modeling in the brain: a practical perspective Magnetic resonance imaging 24:527-539

Henry PG, Tkac I, Gruetter R (2003) ${ }^{1} \mathrm{H}$-localized broadband ${ }^{13} \mathrm{C}$ NMR spectroscopy of the rat brain in vivo at 9.4 $\mathrm{T}$ Magn Reson Med 50:684-692

Jalan R, Olde Damink SW, Deutz NE, Hayes PC, Lee A (2004) Moderate hypothermia in patients with acute liver failure and uncontrolled intracranial hypertension Gastroenterology 127:1338-1346

Jonker R, Deutz NE, Erbland ML, Anderson PJ, Engelen MP (2014) Hydrolyzed casein and whey protein meals comparably stimulate net whole-body protein synthesis in COPD 
patients with nutritional depletion without an additional effect of leucine co-ingestion Clinical nutrition 33:211-220 doi:10.1016/j.clnu.2013.06.014

Kinross JM, Holmes E, Darzi AW, Nicholson JK (2011) Metabolic phenotyping for monitoring surgical patients Lancet 377:1817-1819 doi:10.1016/S01406736(11)60171-2

Kircheis G, Hilger N, Haussinger D (2014) Value of critical flicker frequency and psychometric hepatic encephalopathy score in diagnosis of low-grade hepatic encephalopathy Gastroenterology 146:961-969 doi:10.1053/j.gastro.2013.12.026

Kircheis G, Wettstein M, Timmermann L, Schnitzler A, Haussinger D (2002) Critical flicker frequency for quantification of low-grade hepatic encephalopathy Hepatology 35:357366 doi:10.1053/jhep.2002.30957

Ladep NG et al. (2014) Discovery and validation of urinary metabotypes for the diagnosis of hepatocellular carcinoma in West Africans Hepatology 60:1291-1301 doi:10.1002/hep.27264

Lanz B, Duarte JM, Kunz N, Mlynarik V, Gruetter R, Cudalbu C (2013a) Which prior knowledge? Quantification of in vivo brain 13C MR spectra following 13C glucose infusion using AMARES Magn Reson Med 69:1512-1522 doi:10.1002/mrm.24406

Lanz B, Gruetter R, Duarte JM (2013b) Metabolic Flux and Compartmentation Analysis in the Brain Frontiers in endocrinology 4:156

Lee $\mathrm{KC}$ et al. (2013) A reproducible, clinically relevant, intensively managed, pig model of acute liver failure for testing of therapies aimed to prolong survival Liver international : official journal of the International Association for the Study of the Liver 33:544-551 doi:10.1111/liv.12042

Luiking YC, Poeze M, Deutz NE (2015) Arginine infusion in patients with septic shock increases nitric oxide production without haemodynamic instability Clinical science 128:57-67 doi:10.1042/CS20140343

Luiking YC, Poeze M, Ramsay G, Deutz NE (2009) Reduced citrulline production in sepsis is related to diminished de novo arginine and nitric oxide production The American journal of clinical nutrition 89:142-152 doi:10.3945/ajcn.2007.25765

Mason GF, Rothman DL (2004) Basic principles of metabolic modeling of NMR ${ }^{13} \mathrm{C}$ isotopic turnover to determine rates of brain metabolism in vivo Metabolic engineering 6:75-84

Mirnezami R et al. (2014) Chemical mapping of the colorectal cancer microenvironment via MALDI imaging mass spectrometry (MALDI-MSI) reveals novel cancer-associated field effects Molecular oncology 8:39-49 doi:10.1016/j.molonc.2013.08.010

Mitchell PS et al. (2008) Circulating microRNAs as stable blood-based markers for cancer detection Proceedings of the National Academy of Sciences of the United States of America 105:10513-10518 doi:10.1073/pnas.0804549105

Nicholson JK, Lindon JC, Holmes E (1999) 'Metabonomics': understanding the metabolic responses of living systems to pathophysiological stimuli via multivariate statistical analysis of biological NMR spectroscopic data Xenobiotica; the fate of foreign compounds in biological systems 29:1181-1189 doi:10.1080/004982599238047

O'Grady JG, Schalm SW, Williams R (1993) Acute liver failure: redefining the syndromes Lancet 342:273-275

Olde Damink SW, Dejong CH, Deutz NE, Soeters PB (1997) Decreased plasma and tissue isoleucine levels after simulated gastrointestinal bleeding by blood gavages in chronic portacaval shunted rats Gut 40:418-424

Olde Damink SW et al. (2003) The kidney plays a major role in the hyperammonemia seen after simulated or actual GI bleeding in patients with cirrhosis Hepatology 37:12771285 doi:10.1053/jhep.2003.50221 
Olde Damink SW, Jalan R, Redhead DN, Hayes PC, Deutz NE, Soeters PB (2002) Interorgan ammonia and amino acid metabolism in metabolically stable patients with cirrhosis and a TIPSS Hepatology 36:1163-1171 doi:10.1053/jhep.2002.36497

Romero-Gomez M, Ampuero J (2014) Deciphering the spectrum of low-grade hepatic encephalopathy in clinical practice Gastroenterology 146:887-890 doi:10.1053/j.gastro.2014.02.019

Romero-Gomez $\mathrm{M}$ et al. (2007) Value of the critical flicker frequency in patients with minimal hepatic encephalopathy Hepatology 45:879-885 doi:10.1002/hep.21586

Romero-Gomez M et al. (2010) Variations in the promoter region of the glutaminase gene and the development of hepatic encephalopathy in patients with cirrhosis: a cohort study Annals of internal medicine 153:281-288 doi:10.7326/0003-4819-153-5-20100907000002

Romero-Gomez M, Ramos-Guerrero R, Grande L, de Teran LC, Corpas R, Camacho I, Bautista JD (2004) Intestinal glutaminase activity is increased in liver cirrhosis and correlates with minimal hepatic encephalopathy Journal of hepatology 41:49-54 doi:10.1016/j.jhep.2004.03.021

Sands CJ et al. (2015) Metabolic phenotyping for enhanced mechanistic stratification of chronic hepatitis C-induced liver fibrosis The American journal of gastroenterology 110:159-169 doi:10.1038/ajg.2014.370

Sharma V et al. (2012) Nitric oxide and L-arginine metabolism in a devascularized porcine model of acute liver failure American journal of physiology Gastrointestinal and liver physiology 303:G435-441 doi:10.1152/ajpgi.00268.2011

Swann J et al. (2009) Gut microbiome modulates the toxicity of hydrazine: a metabonomic study Molecular bioSystems 5:351-355 doi:10.1039/b811468d

Szabo G, Bala S (2013) MicroRNAs in liver disease Nature reviews Gastroenterology \& hepatology 10:542-552 doi:10.1038/nrgastro.2013.87

Tsien C et al. (2015) Metabolic and molecular responses to leucine-enriched branched chain amino acid supplementation in the skeletal muscle of alcoholic cirrhosis Hepatology 61:2018-2029 doi:10.1002/hep.27717

Tuvdendorj D, Chinkes DL, Bahadorani J, Zhang XJ, Sheffield-Moore M, Killewich LA, Wolfe RR (2014) Comparison of bolus injection and constant infusion methods for measuring muscle protein fractional synthesis rate in humans Metabolism: clinical and experimental 63:1562-1567 doi:10.1016/j.metabol.2014.09.009

Wilkinson DJ, Atherton PJ, Phillips BE, Greenhaff PL, Smith K (2015) Application of deuterium oxide (D2O) to metabolic research: just D2O it? Depends just how you D2O it! American journal of physiology 308:E847 doi:10.1152/ajpendo.00581.2014 


\section{Figure Legends}

Fig.1 ${ }^{13} \mathrm{C}$ spectroscopy of rat brain at 9.4T using polarization transfer (DEPT), following infusion of $\left[1,6-{ }^{13} \mathrm{C}_{2}\right]$ glucose. A) Axial MR image of the rat brain showing the localization of the $320 \mu$ voxel used for ${ }^{13} \mathrm{C}$ spectroscopy and the position of the ${ }^{1} \mathrm{H}$ quadrature $/{ }^{13} \mathrm{C}$ linearly polarized surface coil. B) Typical localized brain ${ }^{13} \mathrm{C}$ spectrum acquired during 1 hour, 4 hours after the onset of glucose infusion. C) Quantified ${ }^{13} \mathrm{C}$ fractional enrichment of the positions $\mathrm{C} 4, \mathrm{C} 3$ and $\mathrm{C} 2$ of glutamate and glutamine, measured with 10 minutes temporal resolution and averaged over the BDL rat group $(n=7)$.

Fig.2 Summary of the porcine model of acute liver failure (a) and the miRNA results (b). Values are means \pm SE of relative expression $\left(2^{-\Delta \Delta C t}\right)$ for APAP-treated animals $(n=6)$ to both the endogenous control (miR26a) and the time-matched control animals (n=3). $P<0.05$ : * miR122 vs. baseline at -20h; \# miR192 vs. baseline at -20h; $\$$ miR124-1 vs. baseline at $-8 \mathrm{~h}$ (Baker et al. 2015).

Fig.3 Development of overt hepatic encephalopathy in patients with non-TACC haplotype in GLS gene. The critical flicker frequency test with threshold at $39 \mathrm{~Hz}$ enabled the prediction of development of overt hepatic encephalopathy. 


\section{In vivo ${ }^{13} \mathrm{C}$ MRS by polarization transfer:}

A

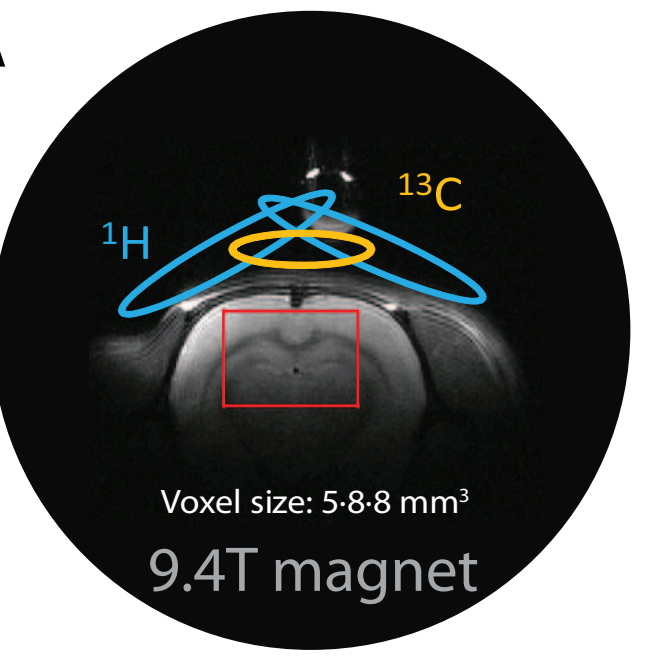

Voxel size: $5 \cdot 8 \cdot 8 \mathrm{~mm}^{3}$

\subsection{T magnet}

B

Glu-C4

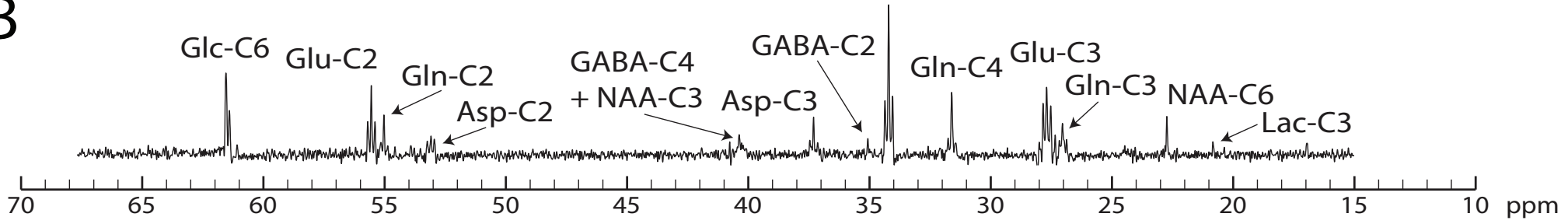

C
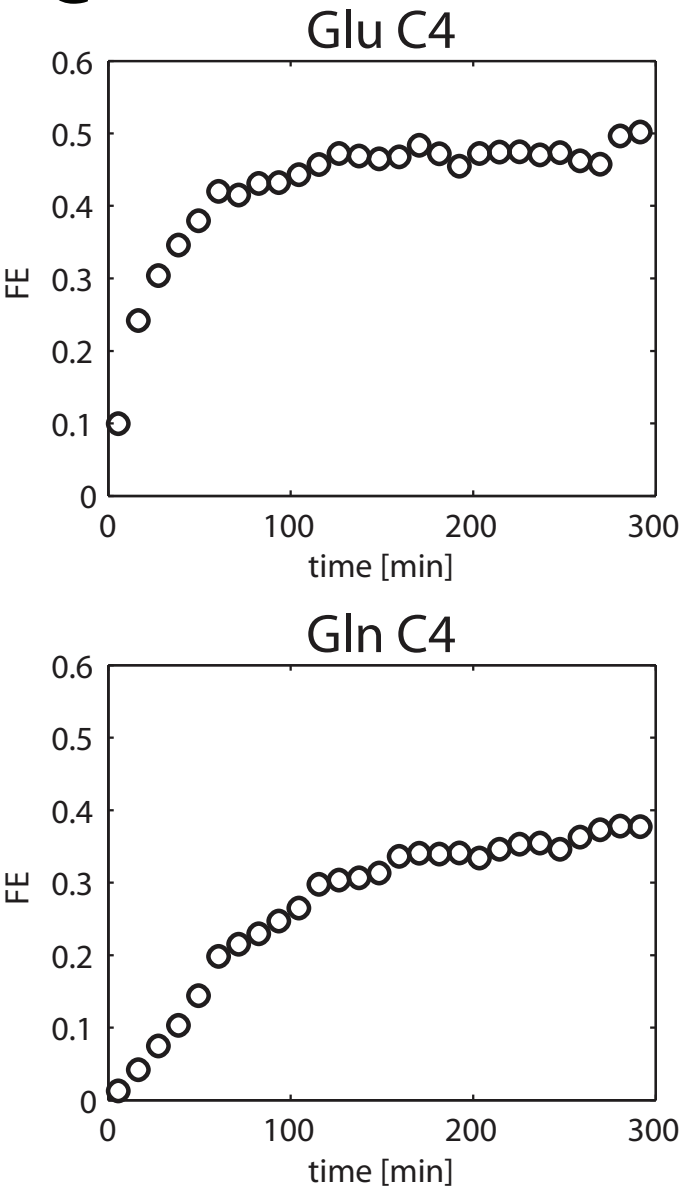

Glu C3

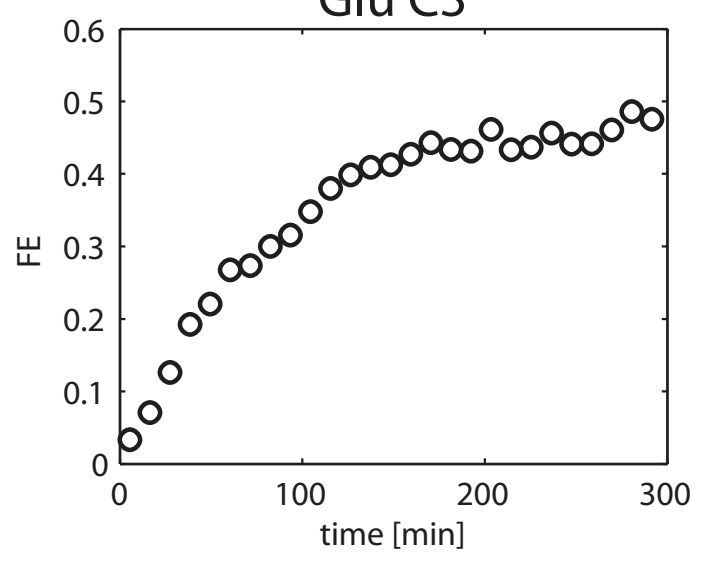

$\mathrm{Gln} C 3$

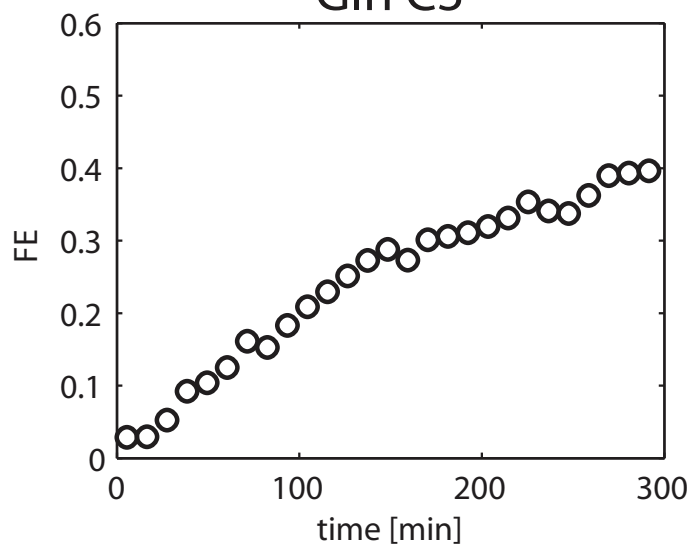

Glu C2

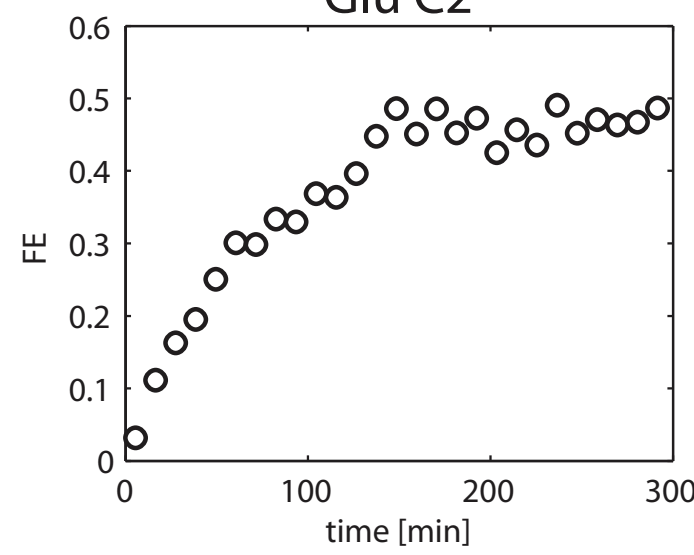

$\mathrm{Gln} C 2$

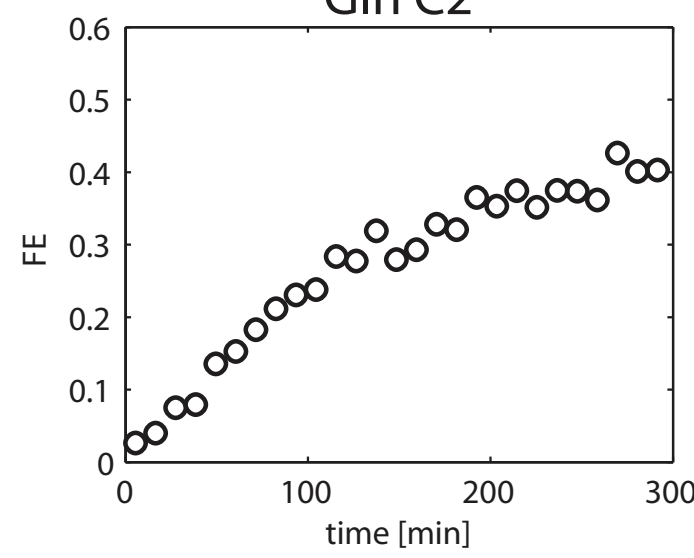




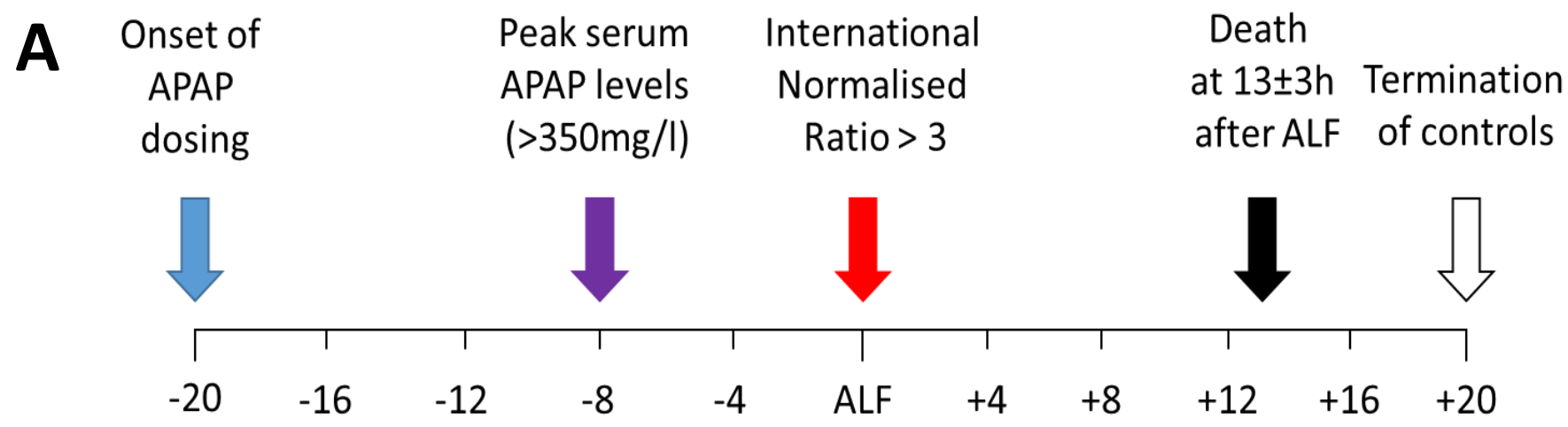

B

\section{Plasma global miRNA levels:}

- Time dependent increase starting $4 \mathrm{~h}$ prior to ALF

- Associated with worsening clinical markers of ALF progression

- Increase found in the protein-bound rather than exosome pool

- Associated with increasing plasma DAMP levels

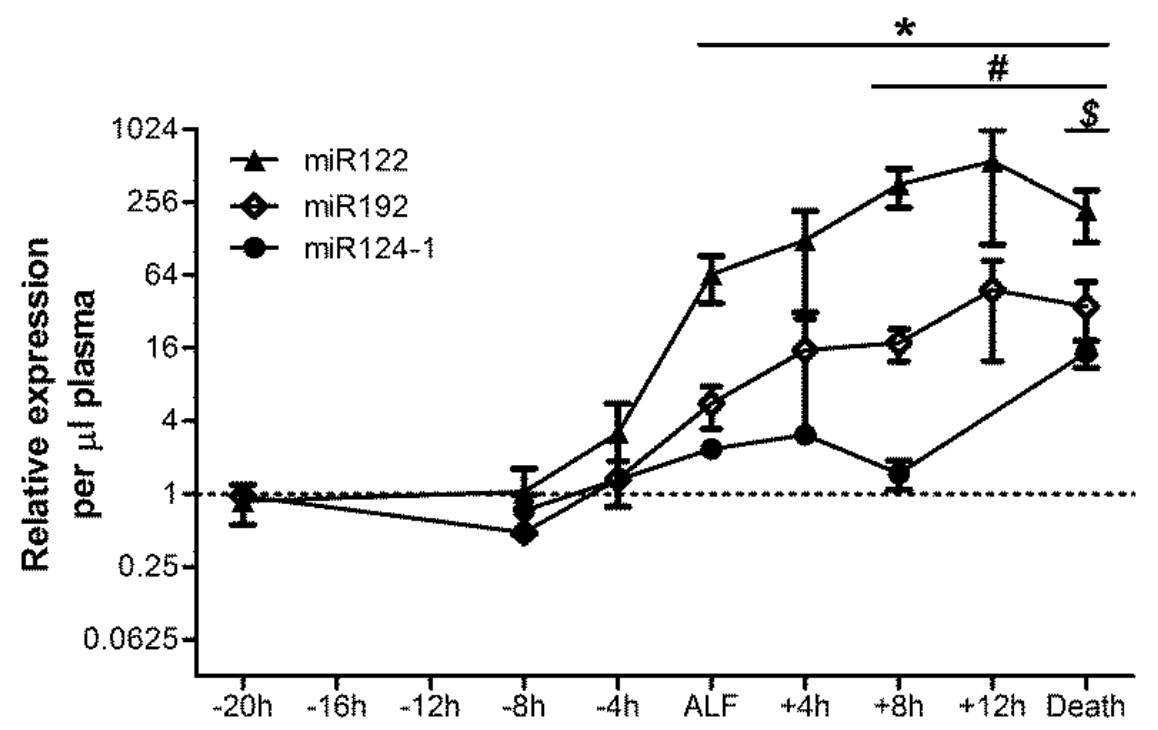

Liver - miR122:

- Increased at onset of ALF

- Associated with increasing INR

\section{Kidney - miR192:}

- Increased 8h after ALF

- Associated with increasing creatinine

Brain - miR124-1:

- Increased pre-terminally

- Associated with decreasing CPP 
NON-TACC HAPLOTYPE

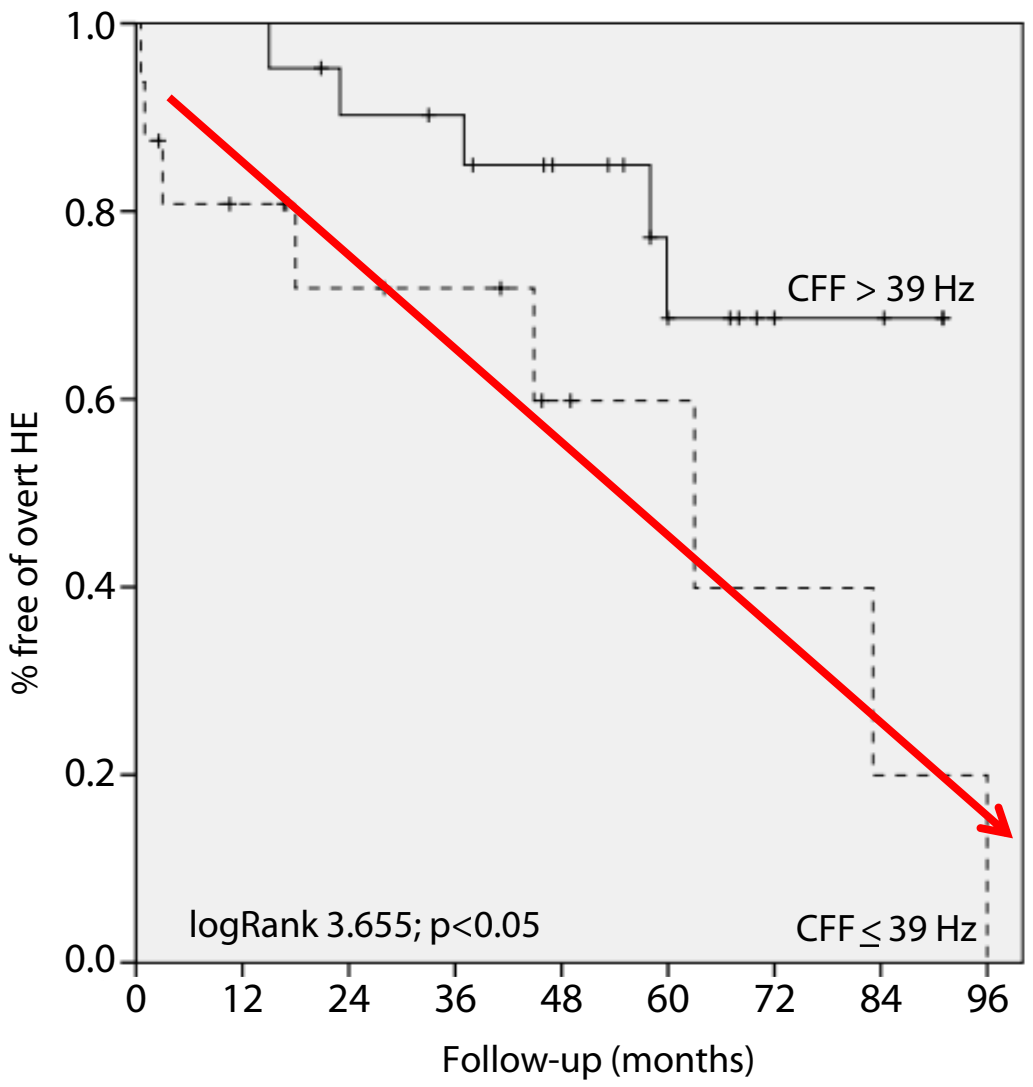

